\title{
INDICE DELL'ANNATA 1993
}

\section{SAGGI}

Declino e innovazione politica nel partito laburista inglese

di Oreste Massari

Minoranze e sicurezza nazionale in Medio Oriente

I primi movimenti socialisti in Europa. Consolidamento organizzativo e mobilitazione politica

di Stefano Bartolini

Lobbying, scambio e definizione degli interessi. Riflessioni sul caso americano di Gigi Graziano

\section{RICERCHE}

Sviluppo organizzativo dei nuovi movimenti sociali e contesto politico di Hanspeter Kriesi

Rilevare la secolarizzazione: indicatori a geometria variabile di Roberto Cartocci

Il debito estero in America Latina. Attori, conflitti e coalizioni di Fabio Fossati

La geometria dello spazio elettorale in Italia di Luca Ricolfi 
L'ascesa dell'Europa meridionale. Centro e periferia nella Comunità europea di Robert Leonardi

\section{NOTE}

Le elezioni nella Cecoslovacchia democratica (19901992)

di Anna Campione

L'identificazione di partito in Italia: due indici a confronto di Stefano Palma

Il nuovo sistema elettorale italiano. Quali opportunità? di Roberto D'Alimonte e A. Chiaramonte

\section{RASSEGNE}

Il neo-istituzionalismo in scienza politica: il contributo della teoria della scelta razionale di Daniela Giannetti

Le tipologie delle politiche pubbliche: una strada senza uscita? di Giliberto Capano

\section{RECENSIONI}

A. Balducci, Disegnare il futuro. Il problema dell'efficacia nella pianificazione urbanistica [Claudio Radaelli]

L. Bonanate (a cura di), Studi internazionali [Luciano Bardi]

R. Boudon, L'ideologie. L'origine des idées reçues [Francesco Zucchini]

L. Cavalli, Governo del leader e regime dei partiti [Sergio Fabbrini]

V. Cesareo (a cura di), La cultura dell'Italia contemporanea [Sonia Stefanizzi] 
A. Colombis, Costruzione e modificazione di concetti. Dalle scale ai fattori [Francesca Rodolfi]

R. D'Amico et al., Manuale di scienza dell'amministrazio$n e$ [Piero Romei]

B. Dente (a cura di), L'efficacia dei poteri locali [Mauro Tebaldi]

G. Di Palma, To Craft Democracies. An Essay on Democratic Transition [Giorgio Alberti]

G. Freddi (a cura di), Medici e Stato nel mondo occidentale. Cultura politica e professionalità medica [Claudio Radaelli]

W. Gormley, Taming the Bureaucracy. Muscles, Prayers, and Other Strategies [Marco Giuliani]

A. Hadenius, Democracy and Development [Leonardo Morlino]

S. Haggard e R.R. Kaufman, The Politics of Economic Adjustment [Fabio Fossati]

E. Hankiss, East European Alternatives [Pietro Grilli di Cortona]

J. Higley e R. Gunther (a cura di), Elites and Democratic Consolidation in Latin America and Southern Europe [Leonardo Morlino]

S.P. Huntington, The Third Wave. Democratization in the Late Twentieth Century [Gianfranco Pasquino]

A. Hyde-Price, European Security Beyond the Cold War [Pierangelo Isernia]

H. Keman (a cura di), Comparative Politics. New Directions in Theory and Method [Stefano Bartolini]

P. Ignazi, Dal Pci al Pds [Carlo Baccetti]

A. La Spina, La decisione legislativa. Lineamenti di una teoria [Giliberto Capano] 
M. Luciani e M. Volpi (a cura di), Referendum. Problemi teorici ed esperienze costituzionali [Pier Vincenzo Uleri]

J.A. Maravall, Stato moderno e mentalità sociale [Francesco Amoretti]

A. Marradi e G. Gasperoni (a cura di), Costruire il dato [Giuseppe Giampaglia]

A. Martinelli (a cura di), International Markets and Global Firms [Alessandro Tonarelli]

K.R. Monroe (a cura di), The Economic Approach to Politics [Daniela Giannetti]

G. Poggi, Lo Stato. Natura, sviluppo, prospettive [Carlo Guarnieri]

M.D. Resnik, Choices [Marco Giuliani]

G. Riccamboni (a cura di), Cittadini e rappresentanza in Europa [Raffaele De Mucci]

R.L. Rothstein (a cura di), The Evolution of Theory in International Relations [Anna Caffarena]

W. Ruding, Anti-Nuclear Movements. A World Survey of Oppositions to Nuclear Energy [Mario Diani]

L. Sebesta, L'Europa indifesa. Sistema di sicurezza atlantico e caso italiano 1948-1955 [Luciano Bozzo]

R. Segatori (a cura di), Istituzioni e potere politico locale [Franca Roncarolo]

D. Sidjanski, L'avenir fédéraliste de l'Europe [Stefano Bartolini]

G. Zincone, Da sudditi a cittadini [Maurizio Ferrera] 